\title{
SOCRATE DE CONSTANTINOPLE ET LES CHRONIQUES
}

L'historien de l'Église Socrate (380/390 - après 439) se distingue de ses collègues en ce qu'il fournit un grand nombre de dates précises. Jusqu'à présent, on suppose en général que Socrate avait utilisé une seule chronique constantinopolitaine à ce but ${ }^{1}$. Pourtant, R.W. Burgess a démontré que l'historien avait accès à plusieurs sources chronographiques. L'édition des Consularia Constantinopolitana dont il s'est servi, n'allait en effet pas audelà de 384 et il lui fallait par conséquent une seconde liste consulaire pour le reste de son ouvrage ${ }^{2}$. Selon lui, Socrate se servait également de la chronique d'Eusèbe de Césarée (ca. 260-339) et de ladite Continuatio Antiochiensis Eusebii, une suite à la chronique d'Eusèbe, allant de 325 à 350 et composée à Antioche ${ }^{3}$. L'hypothèse d'une seule chronique comme source de Socrate n'est ainsi plus défendable.

Il est possible de mener plus loin la déconstruction de cette supposée source unique de Socrate. Nous allons voir que l'historien a utilisé deux listes consulaires (la quatrième édition des Consularia Constantinopolitana jusqu'en 385, et une liste de 388 à 439) et quatre chroniques différentes (la

${ }^{1}$ F. Geppert, Die Quellen des Kirchenhistorikers Socrates Scholasticus (Studien zur Geschichte der Theologie und der Kirche 4). Leipzig 1898, 32-46; W. ELTESTER, Sokrates Scholasticus, in: RE 3.1A (1927) 893-901, 897; Mary Wнітву - M. Whiтву, Chronicon Paschale. 284-628 AD (Translated Texts for Historians 7). Liverpool 1989, xxi; T. D. Barnes, Athanasius and Constantius. Theology and Polities in the Constantinian Empire. Cambridge (Mass.) 1993, 205; G. C. Hansen, Sokrates. Kirchengeschichte (GCS NF 1). Berlin 1995, li-lii; M. WaldrafF, Der Kirchenhistoriker Sokrates. Untersuchungen zu Geschichtsdarstellung, Methode und Person (Forschungen zur Kirchen- und Dogmengeschichte 68). Göttingen 1997, 157; D. Rohrbacher, The Historians of Late Antiquity. Londres 2002, 112; M. Festy, De l'epitome de Caesaribus à la chronique de Marcellin: l'historia romana de Symmaque le Jeune. Historia 52 (2003) 251-255, 252.

${ }^{2}$ R. W. Burgess, The Chronicle of Hydatius and the Consularia Constantinopolitana. Two Contemporary Accounts of the Final Years of the Roman Empire. Oxford 1993. Voir note 17 , où nous suggérons que l'édition utilisée par Socrate se terminait en 385 et non pas en 384, comme le dit R. W. Burgess.

${ }^{3}$ R. W. Burgess, Studies in Eusebian and Post-Eusebian Chronography (Historia Einzelschriften 135). Stuttgart 1999, 148-177. 
chronique d'Eusèbe, la Continuatio Antiochiensis Eusebii, une chronique de 350 à 375 et une chronique de 378 à 422 ou à 439). Notre étude permettra ainsi d'élucider la plupart des erreurs chronologiques de Socrate, qui restaient jusqu'à présent sans explication satisfaisante ${ }^{4}$. Nous avancerons également quelques hypothèses à propos des sources de la chronique de Jérôme (fïn du quatrième siècle), de celle de Marcellinus Comes (sixième siècle) et de la Chronique pascale (début du septième siècle).

Avant d'analyser en détail les consularia (ci-dessous 2) et les chroniques (ci-dessous 3) en question, nous nous devons de clarifier quelques termes et concepts relatifs aux ouvrages chronographiques qui circulaient à cette époque.

\section{DÉfinitions}

Au quatrième et cinquième siècle, on peut observer une distinction entre chroniques et consularia. Elles se différenciaient par la méthode de datation pour l'époque contemporaine.

Les premières contenaient des notices historiques de longueur différente, classées principalement selon les années de règne et les olympiades. Le Chronicon Gallicum a. CCCCLII ${ }^{5}$, les chroniques de Jérôme ${ }^{6}$ et d'Hydace ${ }^{7}$ en sont quelques exemples. Au cinquième siècle certains abandonnaient l'olympiade: Prosper Tiro comptait par exemple à partir de la passion du Christ $^{8}$. Ce développement pourrait être lié au désavouement public de l'olympiade par Théodose II dont on dit qu'il en a abrogé l'usage?.

À côté des chroniques circulaient des consularia. Les listes consulaires ne contenaient que les paires de consuls pour chaque année et éventuellement une brève notice historique. Ces caractéristiques se retrouvent notamment dans les Consularia Constantinopolitana ${ }^{10}$, les Excerpta Latina Barbari $^{11}$ et les Fasti Vindobonenses ${ }^{12}$.

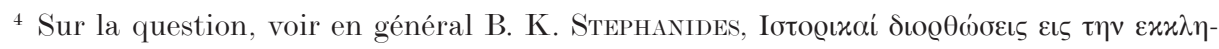

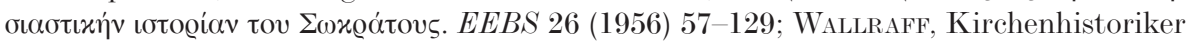
$156-157$.

5 T. Mommsen, Chronica minora: Vol. 1 (MGH Auct. Ant. 9.1). Berlin 1892, 646-662.

${ }^{6}$ R. Helm, Die Chronik des Hieronymus (GCS 47). Berlin ${ }^{3} 1984$.

7 Burgess, Chronicle.

8 Mommsen, Chronica Vol. 1 341-485.

9 Jean Lydos, De Mensibus 4.103. Ni la date ni la raison de cet acte ne sont claires. On peut songer à une manoeuvre religieuse voulant promouvoir un calcul plus chrétien, comme l'ère du monde.

${ }^{10}$ Burgess, Chronicle.

${ }^{11}$ C. Frick, Chronica minora. Leipzig 1892, 184-370.

12 Mommsen, Chronica minora: Vol. 1 274-336.
} 
Il faut attendre le sixième siècle, pour que des écrivains se mettent à brouiller les distinctions entre les deux genres. Ceci s'observe, par exemple, chez Victor Tonnennensis qui utilisait les consuls ${ }^{13}$, ou encore chez Marcellinus Comes qui continuait la chronique de Jérôme en utilisant les consuls et l'indiction comme moyen de datation ${ }^{14}$. Dès cette époque, la datation consulaire devient habituelle parmi les chroniqueurs. Nous en voyons l'effet sur la Chronique pascale (début du septième siècle) $)^{15}$ qui présente à ses lecteurs à la fois les consuls, les années de règne et les olympiades ${ }^{16}$. Force est de constater que l'effacement des distinctions entre les chroniques et les consularia se place plus d'un siècle après que les sources de Socrate furent écrites.

Étant donné que Socrate utilise à la fois des dates consulaires, des olympiades et des années de règne, on peut en conclure qu'il s'est basé simultanément sur des consularia et des chroniques. L'hypothèse d'une source unique chez Socrate présuppose, en effet, que celle-ci aurait dû combiner les caractéristiques propres aux deux genres qui, à son époque, étaient, comme nous l'avons développé, habituellement bien distincts. Ce constat basé sur la définition des genres chronographiques sera confirmé ultérieurement par l'étude des données de Socrate.

\section{Socrate ET Les CONSUlaria}

Comme nous allons voir, Socrate utilisait deux listes consulaires: les Consularia Constantinopolitana dans sa quatrième recension jusqu'en 385, et une liste consulaire perdue de 388 jusqu'à la fin de son histoire.

\subsection{Les Consularia Constantinopolitana}

La première liste consulaire dont se servait Socrate était les Consularia Constantinopolitana. Il s'agit d'une compilation de différentes listes con-

13 T. Mommsen, Chronica minora: Vol. 2 (MGH Auct. Ant. 9.2). Berlin 1892, 184-206.

14 Mommsen, Chronica minora: Vol. 2 37-104. Prosper Tiro (ca. 450) semble le premier.

15 L. Dindorf, Chronicon paschale (CSHB 10-11). Bonn 1832.

16 B. Croke (Chronicles, Annals and 'Consular Annals' in Late Antiquity. Chiron 31 [2001] 291-331) a voulu démontrer l'inexistence de cette distinction. Nous sommes d'accord avec lui que l'opposition idéologique entre consularia, sensés être brefs, anonymes et locaux, et chroniques, considérées comme étant plus étendus et universels, n'est pas justifiée (293-294) et que le vocabulaire de l'époque ne faisait pas de distinction nette entre ces deux genres (301-304). Pourtant, à notre avis, il ne discute pas suffisamment les différences en ce qui concerne la méthode de datation (listes consulaires vs. olympiades et années de règnes), qui distinguent les deux genres pendant presque deux siècles. Ce n'est que par après que les distinctions se brouillent. 
sulaires à partir de la fondation de la république romaine, contenant de brèves notices historiques qui se multiplient à partir du troisième siècle. R.W. Burgess a reconstitué l'histoire de cette liste, dont la première édition était de 337, et la seconde de 342. En 370 circulait une nouvelle édition augmentée de matériau sur Constantinople à partir de 356, ce qui trahit son origine. En 385, l'édition fut mise à jour une quatrième fois ${ }^{17}$. Plus tard, elle était continuée, en plusieurs étapes, jusqu'en 468. À l'encontre de ce qu'écrit R.W. Burgess, il se fait que Socrate utilisa la quatrième édition dès le début de son ouvrage, c.-à-d. à partir de 324 , et non pas à partir de la fin du premier livre $(337)^{18}$.

\subsection{Des consularia de 388 à 439}

La nouvelle liste consulaire utilisée par Socrate ne débutait qu'en 388, comme le trahit un petit trou de deux ans dans ses indications chronologiques, un trou répérable dans les chapitres 12 et 13 du cinquième livre. En 5.12.1-4, Socrate mentionne la naissance du futur empereur Honorius en 384 avec la date consulaire. De plus, il donne les consuls pour 385, l'an de l'élection de Théophile d'Alexandrie. Pour l'année suivante, quand Démophile de Constantinople mourut, il ignore les consuls (5.12.5-8); en effet, l'historien nous dit simplement «dans l'année suivante», sans donner une date consulaire. Les chapitres suivants (5.12.9-11) sont consacrés aux débuts de la guerre (387) contre l'usurpateur Maxime (383-388), dont la fin se voit ultérieurement située en 388 par moyen d'une datation consulaire (5.13.7). Pour le début des préparatifs en 387 (5.12.9-5.13.1), Socrate ne donne pas de dates précises. Puisque l'historien a l'habitude d'offrir pour tout événement une date consulaire, même lorsqu'il souligne qu'il s'agit de l'année suivante (cf. 5.12.5, 6.2.11, 6.20.2), son silence sur les consuls de 386-387 est remarquable. Il suggère que Socrate disposait bel et bien de données historiques pour les années 386-387, mais qu'il en ignorait les

17 Burgess, Chronicle 197 prend 384 pour la fin de cette édition, puisque c'est pour cette année qu'il trouvait le dernier parallèle entre la Chronique pascale, Socrate et les Consularia. Comme les Consularia ne contenaient pas de notice historique pour l'an 385, l'argument de R. W. Burgess ne peut être considéré comme décisif. Nous proposons 385 comme point de chute puisqu'Arcadius et Bauto sont la dernière pair de consuls à être mentionnée par Socrate avant que celui-ci n'ignore les consuls pour les années 386 et 387 (voir ci-dessous 2.2), constituant ainsi le dernier parallèle entre Socrate et les Consularia.

18 Burgess, Chronicle 197. On peut se référer à la liste des parallèles établie par Hansen, Sokrates 405 . 
consuls. Sa liste consulaire précédente s'arrêtait donc en 385 et la nouvelle ne commençait qu'en 388.

Les consularia utilisés par Socrate se distinguent de ceux connus chez d'autres auteurs: d'une part de ceux qu'on retrouve chez Marcellinus Comes et la Chronique pascale, qui se servaient d'une même source, et d'autre part de ceux de la continuation des Consularia Constantinopolitana (voir tableau 1). Ils présentent des traits communs à la fois avec les premiers, provenant de l'empire oriental (cf. 400 et 434) et avec les seconds, d'origine occidentale (cf. 412). Ces divergences ne permettent guère d'effectuer des conclusions sur la liste utilisée par Socrate. Celle-ci était probablement d'origine orientale, car en 434 elle cite le consul d'Orient Aréobindus comme premier. À partir de 421 la pratique est attestée que l'empire de l'Orient et celui de l'Occident citaient chacun comme premier le consul nommé par leur partie de l'Empire ${ }^{19}$. Il semble pourtant qu'il s'agisse d'une liste corrigée sur la base d'une liste occidentale (cf. les consuls de l'an 412).

\begin{tabular}{|c|c|c|c|}
\hline & SOCRATE & $\begin{array}{l}\text { Marc. Com. = } \\
\text { Chron. Pasch. } \\
\text { Liste d'origine orientale }\end{array}$ & $\begin{array}{l}\text { CONS. CONST. CONT. } \\
\text { LISTE D'ORIGINE } \\
\text { OCCIDENTALE }\end{array}$ \\
\hline 400 & $\begin{array}{l}\sum \tau \varepsilon \lambda \dot{i} \chi \omega v o \varsigma \text { xai A } \varrho \eta \lambda \iota \alpha v o \tilde{v} \\
(6.6 .38)\end{array}$ & Stilichone et Aureliano & Stelicone \\
\hline 410 & 1 & Varane solo & Honorio VIII et Varane \\
\hline 411 & 1 & Honorio et Theodosio & Theodosio IIII solo \\
\hline 412 & 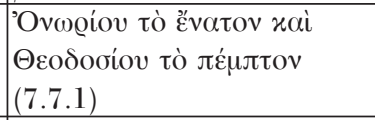 & Theodosio solo & $\begin{array}{l}\text { Honorio VIIII et } \\
\text { Theudosio V }\end{array}$ \\
\hline 413 & $1 /$ & Lucio solo & $\begin{array}{l}\text { pe Honorio VIIII et } \\
\text { Theodosio V/ } \\
\text { Lucio solo }\end{array}$ \\
\hline 434 & 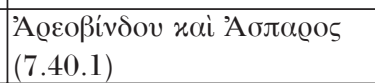 & Areobindo et Aspare & Aspare et Areobindo \\
\hline
\end{tabular}

tableau 1

\section{Socrate ET Les Chroniques}

Avant de déterminer les quatre chroniques dont dépendait Socrate, il nous faut tout d'abord étudier l'usage des années de règne et des olympiades chez celui-ci. Étant donné que ce sont là les caractéristiques spécifiques des chroniques, elles nous aideront à délimiter les sources chronographiques de Socrate.

${ }^{19}$ R. S. Bagnall e.a., Consuls of the Later Roman Empire (Philological Monographs of the American Philological Association 36). Atlanta 1987, 21. 


\subsection{Olympiades et années de règne chez Socrate}

\subsubsection{Les olympiades}

Socrate a l'habitude de marquer les décès des empereurs et les fins des livres (les deux coïncident souvent) par l'année de l'olympiade dans laquelle ils sont apparus (par exemple 1.2.1: Constance II mourut dans la première année du 27lième olympiade). Le troisième livre fait exception avec les morts de Julien (363) et Jovien (364), où aucun des deux événements n'est daté grâce à l'olympiade ${ }^{20}$. L'olympiade qu'un chroniqueur assignait à une certaine année, dépendait évidemment de la date avec laquelle il identifiait la première année de la première olympiade. À l'époque de Socrate se dessinaient deux traditions majeures: celle d'Eusèbe, suivie entre autres par la Chronique pascale, et celle de Jérôme, suivie notamment par Hydace. Précisons qu'Eusèbe a un an d'avance sur Jérôme ${ }^{21}$. Dans le tableau 2 ont été rassemblés tous les passages où Socrate se sert des olympiades, ainsi que les années juliennes correspondantes. Nous y avons ajouté les olympiades qu'il aurait dû y assigner s'il suivait Eusèbe respectivement Jérôme.

\begin{tabular}{|r|c|c|c|c|}
\hline & AN JUL. & SOCRATE & EUs. & HIER. \\
\hline 1.2 .1 & $305^{22}$ & 271.1 & $271.1-2$ & 271.1 \\
\hline 1.40 .3 & 337 & 278.2 & 279.2 & 279.1 \\
\hline 2.47 .5 & 361 & 285.1 & 285.2 & 285.1 \\
\hline 4.38 .7 & 378 & 289.4 & 289.3 & 289.2 \\
\hline 5.26 .5 & 395 & 294.1 & 293.4 & 293.3 \\
\hline 6.23 .7 & 408 & 297.2 & 297.1 & 296.4 \\
\hline 7.20 .13 & 422 & 300.4 & 300.3 & 300.2 \\
\hline 7.48 .8 & 439 & 305.2 & 304.4 & 304.3 \\
\hline
\end{tabular}

tableau 2

À première vue les indications de Socrate sont inconsistantes. D'abord, il suit Jérôme $(1.2 .1,2.47 .5)$; par la suite, il s'en écarte fortement (à partir de 4.38.7). On remarque qu'il ne semble jamais suivre Eusèbe. Si Socrate

${ }^{20}$ Signalons aussi que Socrate ne date pas non plus la mort des empereurs occidentaux à partir de Gratien, c.-à-d. celle de Gratien lui-même et d'Honorius, avec l'olympiade. On y reconnaît la perspective orientale de l'historien.

${ }^{21}$ Il ne nous semble pas exact, comme le font Geppert, Quellen 45 et Hansen, Sokrates 4, de considérer le calcul de Jérôme comme 'correct' et les divergences de Socrate par conséquent comme des 'erreurs'. Le début de l'olympiade était une convention sur laquelle déjà l'antiquité classique ne parvenait pas à s'unir (cf. A. E. SAmuel, Greek and Roman Chronology [Handbuch der Altertumswissenschaft 1, 7], Munich 1972, 189194). 
avait eu recours à une seule chronique, une telle variation aurait été étonnante. Des erreurs se produisent évidemment souvent dans une chronique, mais elles ont tendance à se poursuivre dans l'ensemble de l'ouvrage d'une façon telle que s'imprime une certaine consistance. Par exemple, il manquait dans les Consularia Constantinopolitana deux paires de consuls au début de la liste, manque qui a influencé le dénombrement effectué dans les marges ${ }^{23}$. Un autre exemple est l'index syriaque des lettres festales d'Athanase d'Alexandrie, qui a subi de nombreux remaniements; néanmoins nous pouvons retracer son évolution ${ }^{24}$. Or, il n'en est rien de tel chez Socrate: celui-ci nous présente des données irréconciliables. Il se pourrait donc que Socrate ait utilisé différentes sources dont il aurait simplement copié les indications contradictoires.

En effet, une certaine régularité dans cette inconsistance nous permet de mieux cerner la multiplicité des sources de Socrate. À partir de 378, l'historien présente un écart fixe d'un an par rapport à Eusèbe, respectivement deux ans par rapport à Jérôme. La thèse d'une source commune pour ces indications est plausible. Par ailleurs, à la fin de son livre, l'écart devient deux respectivement trois ans; cela pourrait indiquer un changement de source ou un calcul erroné de la part de Socrate (voir ci-dessous 3.2.4). Les trois premières indications du tableau ne permettent pas encore de mettre en avant des énoncés irréfutables. Cependant, la comparaison avec des sources existantes nous permettra d'assigner chacune de celles-ci à une source différente (voir ci-dessous 3.2.1-3.2.3).

\subsubsection{Les années de règne}

Comme deuxième caractéristique des chroniques nous envisageons les années de règne. Le chroniqueur indique à quelle année de l'empereur régnant correspond chaque entrée dans son ouvrage.

Avant d'étudier l'usage des années de règnes chez Socrate, ajoutons préalablement deux remarques de première importance.

Premièrement, de nombreux chroniqueurs ont l'usage de n'accorder qu’une seule année de règne à chaque année de leur chronique, même si

22 La mort de Constance I est en vérité à situer en 306. Puisque la chronique d'Eusèbe à laquelle Socrate empruntait la donnée la situait en 305 (cf. ci-dessous 3.2.1), l'historien la plaçait sans aucun doute aussi dans cette année-là.

23 Burgess, Chronicle 177.

24 Cf. P. Van Nuffelen, Les lettres festales d'Athanase d'Alexandrie: les «erreurs» chronologiques de l'Index syriaque. Revue des Études Augustiniennes 47 (2001) 85-95, avec littérature antérieure. 
c'est l'année où un empereur mourut et un nouvel empereur lui succéda. La raison en était, d'une part, un souci de clarté dans la disposition visuelle. On notait d'habitude l'année de règne dans le marge et ne voulait pas écrire deux numéros au même endroit. D'autre part, c'était aussi un souci d'exactitude, puisque la somme des années de règne de chaque empereur ne correspondrait plus au total des années de la chronique, quand on compterait à chaque succession certaines années deux fois. Le chroniqueur a alors deux options. Eusèbe choisissait d'assimiler la dernière année de règne de l'empereur à l'année précédant sa mort et la première année de règne de son successeur à l'année de son avènement. Ses successeurs, dont Jérôme est le premier, optèrent pour l'option inverse: la première année du successeur devient l'année qui suit celle de son avènement ${ }^{25}$.

La seconde remarque peut se rattacher à la première. Le chroniqueur assimile souvent l'année de règne à une année julienne ${ }^{26}$. L'année d'un empereur s'écoule alors du premier janvier au trente et un décembre, et non pas de l'anniversaire de son avènement jusqu'à la veille de celui-ci ${ }^{27}$. Dans le cas où le chroniqueur suit la méthode d'Eusèbe, la première année de l'empereur est ainsi une année julienne entière, bien que son avènement puisse tomber au milieu ou vers la fin de cette même année. En revanche, si le chroniqueur suit la méthode de Jérôme, ce sera la dernière année de l'empereur qui sera trop longue.

Néanmoins, cela ne veut pas dire qu'un chroniqueur se voit nécessairement condamné à ne donner que des nombres arrondis pour la durée totale du règne d'un empereur: souvent il fournit un bilan de la durée totale et exacte d'un règne précédant (Jérôme) ou succédant (Eusèbe) le règne complet. Cela montre que le choix d'identifier l'année de règne à une année julienne relève avant tout d'un aspect pratique et ne découle pas toujours d'une ignorance des données exactes.

Il peut donc y avoir une différence entre l'année de règne des chroniqueurs et l'année de règne officielle: la première est une année julienne tandis que la seconde correspond à la période entre le jour de l'avènement

25 Burgess, Studies 42-43, 134-135.

${ }^{26}$ Tout cela évidemment à condition que l'année julienne soit son calendrier de base. S'il se sert de l'année dioclétienne par exemple (Nouvel An le premier septembre), comme l'index syriaque des lettres festales d'Athanase, il assimilera l'année de règne à celle-ci (cf. Van Nuffelen, lettres).

${ }^{27}$ Cf. Eusèbe, Historia Ecclesiastica 2.8.1: Gaius régnait quatre ans (en fait trois ans, dix mois et huit jours), avec les remarques perspicaces d'E. Schwartz - T. Mommsen, Eusebius Werke. Zweiter Band, Dritter Teil: Die Kirchengeschichte (GCS NF 6.3), Leipzig 1909 (= 1995), clxviii. Voir aussi Burgess, Studies 57, 89. 
et l'anniversaire de cette date. Un exemple de la mécompréhension possible de la méthode des chroniqueurs est la controverse établie sur l'année où Constance II devint César. Selon les Consularia Constantinopolitana (a. 361), dont Socrate (2.47.5-6) tire ses informations, Constance mourut le 3 novembre 361. Or, selon Eutrope (10.15.2), il possédait l'imperium pendant trente-huit $\mathrm{ans}^{28}$. On a voulu déduire de cette donnée que Constance était devenu César le 8 novembre $323^{29}$. En effet, en sachant que son jour d'avènement était le 8 novembre (Cons. Const. a. 324), et en acceptant l'hypothèse que les sources citées suivaient le calcul officiel des années de règnes, les savants ont assimilié la trente-huitième année de Constance avec l'année de règne allant du 8 novembre 360 jusqu'au 7 novembre 361 , ce qui les a conduit à proposer comme première année de règne la période du 8 novembre 323 jusqu'au 7 novembre 324. Pourtant, un avènement le 8 novembre 323 est en contradiction avec les sources qui donnent explicitement la date de l'avènement. Selon les Consularia Constantinopolitana (a. 324) ce fut le 8 novembre 324 que Constance II devint César. Jérôme (Chron. a. 324) ne donne que l'année 324. Les papyrus placent la prise de l'imperium de Constance entre le 30 août 324 et le 24 juillet $325^{30}$. Toutefois, la difficulté disparaît dès qu'on prend conscience du fait que la trente-huitième année que nous donne Eutrope, ne correspond pas à une année de règne officielle (du 8 novembre au 7 novembre), mais à une année julienne. La trente-huitième année étant assimilée à l'année julienne 361 , la première année de

${ }^{28}$ Cf. aussi Ammien Marcellin 21.15.2 (mais tricesimo octavo anno est une conjecture); Ps.Denys, Chronique (ed. J. B. Снавот, Incerti auctoris chronicon Pseudo-Dionysianum vulgo dictum [CSCO 121] 131.22-24). Selon Epitome de Caesaribus 42.17, Constance II régnait trente-neuve ans. C'est en tout cas faux.

${ }^{29}$ Cf. H. F. Clinton, Fasti romani. The civil and literary chronology of Rome and Constantinople from the Death of Augustus to the Death of Justin II. Oxford 1845, Vol. 1 446; J. Den Boeft e.a., Philological and Historical Commentary on Ammianus Marcellinus XXI. Groningen 1991, 232-235 (avec littérature); A. DemandT, Die Spätantike: römische Geschichte von Diocletian bis Justinian, 284-565 n. Chr. (Handbuch der Altertumswissenschaft 3,6). Munich 1989, 92 n. 74.

${ }^{30}$ P. Oslo II 44 (24 juillet 325) donne les années de règne 19 (Constantin le Grand), 9 (Crispus et Constantin II) et 1 (Constance II). Puisque le comput égyptien comptait comme première année la période entre l'avènement et Nouvel An ( 1 Thot $=29$ ou, dans les années bissextiles, 30 août), et faisait ensuite coïncider l'année régnale avec l'année égyptienne, l'avènement de Constance doit se placer entre le 30 août 324 et 24 juillet 325. Voir sur cette question A. Chastagnol, La datation par années régnales égyptiennes à l'époque constantinienne, in: AION. Le temps chez les Romains (Caesarodunum 10bis). Paris 1967, 221-238; R. Bagnall - K. Worp, The Chronological Systems of Byzantine Egypt (Studia Amstelodamensia ad epigraphicam, ius antiquum et papyrologiam pertinentia 8 ). Zutphen 1978, 38-40. 
Constance II correspond à 324. C'est donc bien le 8 novembre 324 que Constance assuma l'imperium.

Revenons désormais à Socrate. Tout comme pour les olympiades, des inconsistances dans le système des années de règne peuvent nous donner des renseignements importants sur un changement de source chez cet auteur.

Dans le tableau suivant (tableau 3) nous résumons les indications de Socrate concernant les années de règne. Nous présentons dans la deuxième colonne les passages de Socrate où il date selon les années de règne, et dans la colonne suivante nous écrivons l'année du règne donnée par Socrate et l'année julienne correspondante (par exemple $7=312$ ). L'année julienne est en principe celle que Socrate donne lui-même (il donne d'habitude également une date consulaire). La quatrième colonne indique la première année du règne qui résulte des indications de Socrate (par exemple 1=306), et la dernière donne sa source pour ce passage.

\begin{tabular}{|l|l|l|l|l|}
\hline EMPEREUR & SOCRATE & ANNÉE DE RĖGNE & DÉBUT & SOURCE \\
\hline Constantin I & 1.2 .7 & $7=312$ & $1=306$ & $\begin{array}{l}\text { Eus. Chron. a. 304-305 } \\
\text { (cf. ci-dessous 3.2.1) }\end{array}$ \\
\hline & 1.13 .13 & $19=325$ & $1=307$ & «Actes» de Nicée $^{31}$ \\
\hline & 1.16 .1 & $20=325$ & $1=306$ & $\begin{array}{l}\text { Eus. Chron. a. 325 } \\
\text { (cf. ci-dessous 3.2.1) }\end{array}$ \\
\hline & 1.28 .2 , cf 1.37 .1 & $30=335$ & $1=306$ & Eus. Vita Constantini 4.40.1 \\
\hline total (Aug.) & 1.40 .3 & $31=337$ & $1=307$ & Eutr. 10.8.2 \\
\hline Constance II & 2.8 .5 & $5=341$ & $1=337$ & $\begin{array}{l}\text { Cont. Ant. (cf. ci-dessous } \\
3.2 .2)\end{array}$ \\
\hline total (Caesar) & 2.47 .6 & $38=361$ & $1=324$ & Eutr. 10.15.2 \\
\hline total (Aug.) & 2.47 .6 & $25=361$ & $1=337$ & Cf. ci-dessous 3.2.3 \\
\hline $\begin{array}{l}\text { Julien } \\
\text { (tot. Caesar) }\end{array}$ & 3.21 .18 & $7=363$ & $1=357$ & Eutr. 10.16.2 \\
\hline total (Aug.) & 3.21 .18 & $3=363$ & $1=361$ & Cf. ci-dessous 3.2.3 \\
\hline $\begin{array}{l}\text { Jovien } \\
\text { total (Aug.) }\end{array}$ & 3.26 .5 & 7 mois & & Eutr. 10.18.2 \\
\hline $\begin{array}{l}\text { Valentinien I } \\
\text { total (Aug.) }\end{array}$ & 4.31 .6 & $11=375$ & $1=365$ & Cf. ci-dessous 3.2.3 \\
\hline total (Aug.) & 4.38 .11 & $13=375$ & $1=363$ & \\
\hline
\end{tabular}

${ }^{31}$ H.-G. OpITz, Die Zeitfolge des arianischen Streites von den Anfängen bis zum Jahre 328. Zeitschrift für die Neutestamentliche Wissenschaft 33 (1934), 131-159, 154 n. 119. Il faut corriger une erreur dans l'apparat de l'édition de G. C. Hansen: à la page 51 il faut

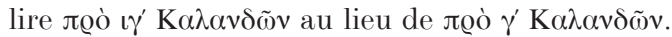

${ }^{32}$ La dépendance d'Eutrope élimine la raison d'être de l'hypothèse de GEPPERT, Quellen 43 qui pensait que Socrate utilisait une chronique dans laquelle il manquait les années 356 et 357, pour réconcilier les 7 ans du césarat (impliquant un avènement en 357) avec un avènement réel en 355 . 


\begin{tabular}{|l|l|l|l|l|}
\hline EMPEREUR & SOCRATE & ANNÉE DE RĖGNE & DÉBUT & SOURCE \\
\hline Valens & 4.38 .11 & $16=378$ & $1=363$ & Cf. ci-dessous 3.2.4 \\
\hline $\begin{array}{l}\text { Gratien } \\
\text { total (Aug.) }\end{array}$ & $5.11 .9^{33}$ & $\begin{array}{l}15=383 \\
(\text { ou } 17=383)\end{array}$ & $\begin{array}{l}1=369 \\
(1=367)\end{array}$ & Cf. ci-dessous 3.2.4 \\
\hline $\begin{array}{l}\text { Théodose I } \\
\text { (total) }\end{array}$ & 5.26 .6 & $16=394$ & $1=379$ & Cf. ci-dessous 3.2.4 \\
\hline $\begin{array}{l}\text { Arcadius } \\
\text { total (Caesar) }\end{array}$ & 6.23 .7 & $13=395$ & $1=383$ & \\
\hline total (Aug.) & 6.23 .7 & $14=408$ & $1=395$ & Cf. ci-dessous 3.2.4 \\
\hline
\end{tabular}

tableau 3

De ce tableau nous pouvons tirer deux conclusions provisoires.

(1) Pour les règnes de Constantin (324-337) et de Constance II (337-361), Socrate a utilisé plusieurs sources, consistant en des chroniques et d'autres ouvrages, tels que la Vie de Constantin d'Eusèbe, la chronique du même, des "Actes de Nicée», Eutrope et la Continuatio Antiochiensis Eusebii. Ses données chronologiques proviennent donc de nombreuses sources et sont intégrées dans le trame consulaire. En ce qui concerne les règnes suivants, nous ignorons les sources de Socrate, à l'exception d'Eutrope, dont il a également fait usage pour le règne de Julien.

(2) Les premières indications pour le total des années de règne de Constantin à Jovien se basent sur Eutrope. Dès le règne de Valentinien (364-375) et de Valens (364-378), lorsque l'ouvrage d'Eutrope se termine, Socrate nécessite une autre source. Il semble qu'il s'en est servi de deux. L'historien donne deux indications contradictoires pour la durée du règne de Valentinien, onze et treize ans $(4.31 .6,4.38 .11)$, ce qui nous permet d'y déceler un changement de source (voir ci-dessous 3.2.3).

33 Le texte grec lit 15 ans au lieu de 17 , ce qui donnerait $1=369$. Le règne de Gratien débutait pourtant en 367, ce qui demande un total de 17 ans. La traduction arménienne de Socrate (ed. M. TER-Movsesean, Vienne 1897, 432) donne le chiffre exacte de 17 ans. Il est donc possible qu'il faut lire $\iota \zeta^{\prime}$ au lieu de $\iota \varepsilon^{\prime}$ dans le texte grec. D'autre part, le remaniement de la traduction arménienne (ledit Socrates Minor, voir R. W. Thomson, The Armenian Adaptation of the Ecclesiastical History of Socrates Scholasticus [Hebrew University Armenian Studies 3], Louvain 2001) donne le nombre de 15 ans, tout comme le texte grec. Deux possibilités se présentent alors: ou bien le nombre de 17 ans est une corruption dans les manuscrits de la traduction arménienne, et Socrate a alors écrit 15 ans; ou bien le nombre de 15 ans dans Socrates Minor est une corruption identique à celle qui se serait produit dans le texte grec. Dans ce cas, seule la traduction arménienne conserve le nombre original de 17 ans. Il faut avouer que la seconde possibilité est la moins probable. 


\subsection{Les chroniques utilisées par Socrate}

Les erreurs dans les olympiades et les années de règne nous ont fourni suffisamment de données pour pouvoir déceler les chroniques dont Socrate s'est servi. Passons à leur détermination.

\subsubsection{La chronique d'Eusèbe}

R.W. Burgess nous a fourni une reconstruction de la dernière partie de la chronique d'Eusèbe, dont la seconde édition couvrait l'histoire mondiale jusqu'en 325. Socrate l'a utilisée à trois occasions (tableau 4) ${ }^{34}$.

\begin{tabular}{|l|l|}
\hline Socrate & Eus. Chron. \\
\hline 1.2 .1 & a. $304-305$ \\
\hline 1.2 .7 & a. 312 \\
\hline $1.16 .1^{35}$ & a. 325 \\
\hline
\end{tabular}

tableau 4

À 1.2.1 Socrate donne Ol. 271.1 comme la date de la mort de Constance I (située en 305) en olympiades, ce qui devrait être Ol. 271.2 selon le calcul eusébien. Pourtant, sa provenance de la chronique d'Eusèbe est assurée par le fait que, chez Eusèbe, Ol. 271 ne contient que trois ans avec Ol. 271.1 couvrant les années 304-305 ${ }^{36}$. L'évêque de Césarée situe la mort de Constance I dans ces années-là. On peut donc en déduire que Socrate n'a pas vu qu'il y avait une erreur dans sa source.

\subsubsection{La Continuatio Antiochiensis Eusebii}

La deuxième chronique utilisée par Socrate était la Continuatio Antiochiensis, une continuation de la chronique d'Eusèbe de 325 jusqu'en 350 composée à Antioche. Il est probable que Socrate ait utilisé la chronique d'Eusèbe dans un même manuscrit avec la Continuatio. R.W. Burgess a montré la dépendance de Socrate à cet ouvrage ${ }^{37}$. Nous complétons son inventaire dans le tableau suivant (5).

34 Ces références sont à inclure dans l'apparat de Burgess, Studies.

35 Socrate a copié deux fois, en 1.2 .7 et 1.16 .1 , les années de règne que donne d'Eusèbe (cf. tableau 3).

36 Burgess, Studies 40-41, 62-63. Cf. R. W. Burgess, The Dates and Editions of Eusebius' chronici canones and historia ecclesiastica. Journal of Theological Studies 48 (1997) 471-504, 477 n. 19.

37 Burgess, Studies 119-122. 


\begin{tabular}{|l|l|l|l|}
\hline Socrate & ConT. ANT. & SocRATE & \multicolumn{1}{c|}{ ConT. ANT. } \\
\hline 1.16 .1 & a. 325, a. 330 & 2.8 .2 & a. $341^{38}$ \\
\hline 1.17 .1 & a. 327 & 2.8 .5 & a. $327,329,330,341$ \\
\hline 1.18 .4 & cf. a. 327 & 2.10 .1 & cf. a. 339 \\
\hline $1.39 .1-5$ & cf. a. 337 & $2.26 .9-10$ & a. $330,341,344^{39}$ \\
\hline 1.40 .3 & a. $337^{40}$ & & \\
\hline
\end{tabular}

tableau 5

\subsubsection{Une chronique de 350 à 378}

Une nouvelle chronique est utilisée par Socrate pour la période de 350 à 375 . Les olympiades et les années de règne se distinguent en effet clairement des données antérieures et postérieures. L'an 361 est marqué comme Ol. 285.1 (2.47.3), ce qui suggère l'utilisation d'un calcul hiéronymien, la méthode eusébienne donnant 285.2 pour cette même année (voir tableau 2). L'indication suivante, celle de l'année de mort de Valens 378, abandonne ce système au profit d'un calcul avec un surplus de deux années par rapport à Jérôme; on peut donc en déduire qu'elle provient d'une autre source. La durée du règne de Valentinien est indiquée comme étant de onze ans, ce qui rejoint le calcul de Jérôme (4.31.6, a. 375). Un peu plus loin, Socrate accorde treize ans à Valentinien et seize ans à Valens (4.38.11). Ces derniers chiffres présentent effectivement un surplus de deux ans, inexplicable, si ce n'est par l'argument du changement de source (voir ci-dessous 3.2.4). Nous envisageons done que Socrate possédait une chronique qui allait à peu près de 350 (date à laquelle la Continuatio se terminait) à 378 (la date pour laquelle une nouvelle source doit être acceptée à cause des années de règnes contradictoires pour Valentinien).

${ }^{38}$ Socrate commet une erreur en attribuant dix ans à la construction de l'église d'Antioche.

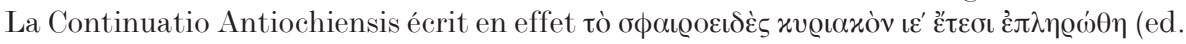
Burgess, Chronicle: cf. Jérôme, Chronique a. 342; Théophane p. 36.29-31 [de Boor]; Chronicon 724 p. 130.21-24 [CSCO 6]; Michel le Syrien, Chronique 7.4 [Chabot]). Il est probable que l'epsilon soit tombé dans le manuscrit de Socrate, ou que l'historien ait simplement mal lu sa source (voir aussi n. 40).

${ }^{39}$ L'aperçu des évêques d'Antioche provient probablement des données de la continuatio. Il n'y a pas lieu de supposer que Socrate ait utilisé ici une liste épiscopale d'Antioche: sa connaissance des évêques antiochiens est trop lacunaire pour qu'on puisse accepter qu'il aurait eu devant lui une liste complète des occupants du siège dès le début du quatrième siècle (c'était l'idée de GEPPERT, Quellen 55, qui donne un tableau avec les données de Socrate).

${ }^{40}$ Socrate écrit Ol. 278.2 au lieu de Ol. 279.2. Il a sans doute lu une H (8) au lieu de la $\Theta$ (9) du manuscrit. 
Cette chronique suivait les principes hiéronymiens: en accordant un règne de onze ans à Valentinien, elle assimile sa première année à l'année qui suit son avènement, en 365, tout comme Jérôme. Pour Constance II et Julien la question est moins claire. En effet, en accordant vingt-cinq ans au premier, Socrate situe son avènement en 337, alors que selon les principes hiéronymiens ce devrait être 338. Nous pouvons l'expliquer si nous acceptons préalablement que cette chronique applique les principes hiéronymiens dès le règne de Constance II, c.-à-d. à partir de 337 (y inclus), en comptant jusqu'en $361^{41}$. Les trois années accordées à Julien (361-363) ne peuvent s'expliquer que par le fait que Socrate, ou sa source, ont compté l'an 361 deux fois (une fois comme dernière année de Constance II, la deuxième fois comme première de Julien), ce qui irait à l'encontre de la pratique habituelle des chroniqueurs. Il n'est pas non plus à exclure l'hypothèse selon laquelle cette donnée serait issue d'une autre source.

Si l'on passe en revue tous les passages à caractère chronologique pour la période $350-378$, on peut constater de nombreux parallèles avec la chronique de Jérôme et avec la Chronique pascale (tableau 6$)^{42}$.

\begin{tabular}{|c|c|c|}
\hline SOCRATE & HIER. & CHRON. PASCH. \\
\hline $2.28 .15-20^{43}$ & & p. 539.20-540.5 \\
\hline $2.28 .21-22$ & [a. 351$]$ & p. $540.8-12$ \\
\hline $2.32 .1-9^{44}$ & $\lceil$ a. 352$]$ & p. $541.10-13$ \\
\hline $2.32 .10-11$ & [a. 353$]$ & \\
\hline
\end{tabular}

${ }^{41}$ Le changement d'un système à l'autre, sans adapter tous les règnes précédents implique nécessairement que le chroniqueur accorde une année supplémentaire à un empereur. Cf. l'intervention de Jérôme, quand il passe du calcul eusébien au calcul hiéronymien (Burgess, Studies 42-43).

${ }^{42}$ Pour ces parallèles, voir aussi Hansen, Sokrates ad loc.

${ }^{43}$ Socrate dépend ici d'une source supplémentaire, soit Julien, Discours 1.31d-32a soit Thémistius, Discours 2.37b-38b - ou les deux en même temps.

${ }^{4}$ Socrate a combiné plusieurs sources, qui donnaient des dates différentes pour la mort de Magnence: Chronique Pascale a. 353, le 10 août 353; Consularia Constantinopolitana a. 353, le 11 août 353. Socrate pouvait aussi lire à Consularia Constantinopolitana a. 350 que Magnence fut proclamé empereur le 18 janvier 350 et chez Eutrope 10.12.2 qu'il régnait 3 ans et 7 mois, situant ainsi sa mort le 18 août 353. Face à ces données inconsistantes, Socrate propose le compromis comme quoi Magnence fut mort «vers le

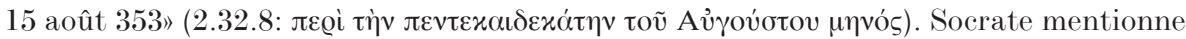
également deux frères de Magnence, alors qu'il n'y en avait en vérité qu'un seul: l'un, c'est le frère anonyme que tue Magnence dans la Chronique pascale (p. 541.10-13 [Bonn]), l'autre, c'est Décentius, qui se suicide après la mort de Magnence selon Consularia Constantinopolitana a. 353. Voir Hansen, Sokrates 148. 


\begin{tabular}{|l|l|l|}
\hline SOCRATE & HiER. & Chron. PASCH. \\
\hline $2.33^{45}$ & a. 352 & \\
\hline $2.34 .1-5$ & ef. a 352 et 354 & p. $541.15-17$ \\
\hline $2.47 .3-4^{46}$ & {$[$ a. 361$]$} & p. $545.7-12$ \\
\hline $3.2 .10^{47}$ & cf. a. 362 & p. $546.6-10$ \\
\hline
\end{tabular}

45 Socrate est, avec la chronique de Jérôme, une des rares sources à mentionner cette révolte des juifs dans les années 352-353: voir aussi Aurélius Victor 42.11; Théodoret, Historia ecclesiastica 4.22.36. Cf. P. SснӓғER, Der Aufstand gegen Gallus Caesar, in: Tradition and Re-interpretation in Jewish and Early-Christian Literature. Essays in Honour of J.C.H. Lebram (Studia Postbiblica 36). Leyde 1986, 184-201; J. ARcE, La rebelión de los Judíos durante el gobierno de Constancio Galo Cesar: 353 d.C., Athenaeum 65 (1987) 109-125; Joshua Schwartz, Gallus, Julian and Anti-Christian Polemic in Pesikta Rabbati. Theologische Zeitschrift 46 (1990), 1-19.

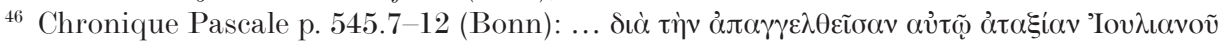

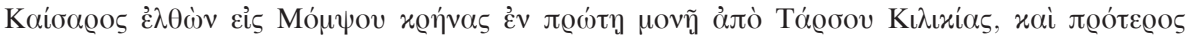

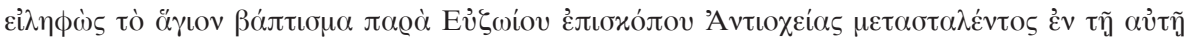

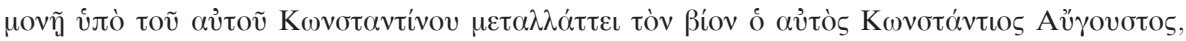
$\mu \eta v i \mathrm{i} \omega$...

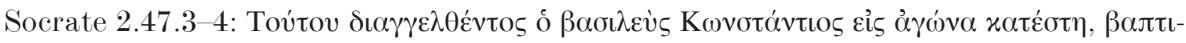

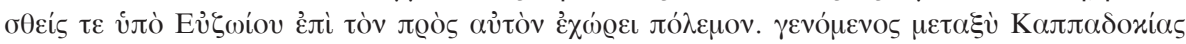

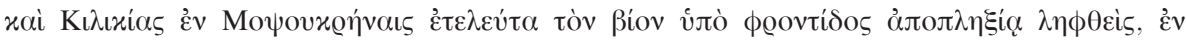

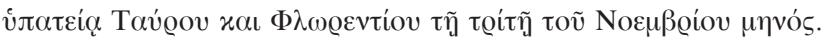

Les deux sources sont les seules à faire état d'un baptême de Constance II par Euzoios. La Chronique pascale ne peut pas avoir copié Socrate, qui ne situe pas Mopsoucrénas par rapport à Tarsos, ni ne dit l'historien qu'Euzoios était appelé d'Antioche. Socrate peut avoir pris le renvoi à Euzoios à Athanase, De Synodis 31.3, un ouvrage qu'il connaissait.

Socrate a sans doute pris la date de la mort de Constance à Consularia Constantinopolitana a. 361 .

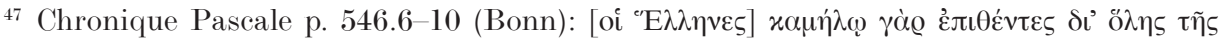

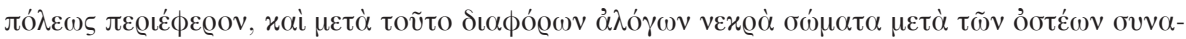

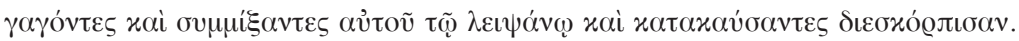

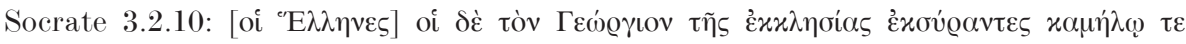

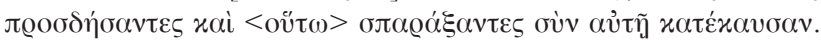

Cf. H.C. Brennecke, Christliche Quellen des Ammianus Marcellinus. Zeitschrift für Antikes Christentum 1 (1997) 226-250, 244-247 qui montre que Socrate et la Chronique pascale dépendent d'une source commune (voir aussi H. C. Brennecke, Studien zur Geschichte der Homöer des Ostens bis zum Ende der homöischen Reichskirche [Beiträge zur historischen Theologie 73]. Tübingen 1988, 116-118).

En revanche, que cette source soit l'historien homéen anonyme (sur cet auteur voir ci-dessous), qui aurait composé une histoire ecclésiastique des persécutions dioclétiennes jusqu'à la mort de Valens, comme le prétend M. Brennecke, est peu probable. Nous venons de montrer que la source en question était une chronique. M. Brennecke a aussi tendance a surestimer l'étendu de cette histoire homéenne (cf. F. WinkELMAnn, Zur nacheusebianischen christlichen Historiographie des 4. Jahrhunderts, in: Polypleuros nous. Miscellanea für Peter Schreiner [Byz. Archiv 19]. Leipzig 2000, 405-414, 414). 


\begin{tabular}{|c|c|c|}
\hline SOCRATE & HIER. & CHRON. PASCH. \\
\hline 3.13 .4 & & ef. p. $549.6-10$ \\
\hline 4.1 .2 & cf. a. 364 & \\
\hline 4.8 .8 & a. 373 & p. 556.11 \\
\hline 4.9 .5 & & p. 556.18 \\
\hline $4.10^{48}$ & & p. $557.7-9$ \\
\hline 4.11 .1 & & \\
\hline 4.31 .6 & a. 375 & \\
\hline 4.35 .1 & a. 377 & \\
\hline
\end{tabular}

tableau 6

Bien que les liens entre Socrate, Jérôme et la Chronique pascale soient généralement peu manifestes, la dépendance à une source commune est rendue probable par des parallèles clairs, tels qu'on en trouve en 2.32.1-9, 2.47.3-4, 4.10 (avec la Chronique pascale), 2.33 (avec Jérôme) et 3.2.10 (avec Jérôme et la Chronique pascale).

La Chronique pascale du septième siècle ne connaissait pas l'histoire de Socrate, même pas à travers l'histoire de Théodore Lecteur (6ième siècle), qui copiait celui-ci ${ }^{49}$. Les parallèles entre la Chronique pascale et Socrate proviennent done d'une source commune. Bien que la Chronique pascale semble avoir utilisé les Consularia constantinopolitana ${ }^{50}$, les parallèles entre celle-là et Socrate ne peuvent être expliqués par l'usage commun de ces consularia. Une autre source doit alors se trouver à l'origine de ces notices communes.

Le cas de Jérôme est bien plus compliqué. Rien ne permet d'affirmer que Socrate l'ait consulté directement. Les deux auteurs ont plutôt plusieurs sources en commun. Jérôme utilisait une version des Consularia Constantinopolitana (sans doute l'édition de 370) ${ }^{51}$, tout comme le faisait Socrate. Les [] figurant dans le tableau 6 indiquent les endroits où les parallèles entre Jérôme et Socrate sont probablement dus à l'usage de cette

Le fait que nous pouvons assigner ces passages à la chronique commune de Socrate, Jérôme et la Chronique pascale, rend probable qu'il faut abandonner l'idée même d'une telle histoire homéenne à partir de la persécution dioclétienne jusqu'à la mort de Valens, proposée par J. Bidez, Philostorgius. Kirchengeschichte (GCS). Berlin ${ }^{2} 1972$, 201-241. Burgess, Studies avait par ailleurs déjà pu montrer que les fragments jusqu'en 350, attribués auparavant à cette supposée histoire ecclésiastique anonyme, appartiennent en vérité à une chronique qu'il a baptisé Continuatio Antiochiensis Eusebii.

48 Socrate et la Chronique pascale situent en 366 la naissance de Valentinien II, le fils de Valentinien I (364-375), alors qu'il s'agit du fils de Valens (364-378).

${ }^{49}$ Cf. Whiтву - Whitbi, Chronicon paschale $\mathrm{xv}-\mathrm{xv}$ iii.

${ }^{50}$ Whiтву - Whiтву, Chronicon paschale xvii.

${ }^{51}$ Cf. Burgess, Chronicle 196. 
source commune. Cependant, il reste encore des parallèles qui ne peuvent pas s'expliquer par le biais de cet usage commun des Consularia. La complexité de la discussion s'accroît par le fait qu'on assigne au moins un des passages de Jérôme qui trouve un parallèle chez Socrate (Jérôme, Chronique a. 352, Socrate 2.33, comparez aussi Jérôme, Chronique a. 352, 354 et Socrate 2.34.1-5) à la dite Kaisergeschichte, un bref ouvrage sur les empereurs romains jusqu'à la première moitié du quatrième siècle ${ }^{52}$. Or, si l'on accepte que Socrate utilisait la Kaisergeschichte ${ }^{53}$, le passage en question peut donc provenir de cette source et non pas d'une chronique commune. Cependant, la Kaisergeschichte n'allant sans doute pas au-delà de 357, elle ne peut pas rendre compte des autres parallèles entre Socrate et Jérôme, ni, en aucun cas, des parallèles entre ces deux auteurs et la Chronique pascale. Il est donc permis de conclure que Jérôme et Socrate ont consulté une source commune, à part des Consularia Constantinopolitana et (peut-être) de la Kaisergeschichte.

L'hypothèse d'une chronique commune entre Socrate, Jérôme et la Chronique pascale pour les années 350-378 nous semble done défendable. Il est déplorable qu'une reconstruction de cette source commune n'est pas envisageable, vu que les parallèles y sont trop peu nombreux. Néanmoins, les conclusions suivantes sont permises.

(1) La chronique que nous proposons comme source probable de Jérôme, Socrate, et la Chronique pascale a deux traits en commun avec la chronique de Jérôme: elle se terminait en 378 et utilisait le calcul hiéronymien pour les olympiades (cf. tableau 2). Cela suggère que Jérôme aurait emprunté ces éléments à cette source. Ce constat a deux conséquences. Tout d'abord, nous ne devons pas surestimer l'originalité de Jérôme. Le calcul «hiéronymien» ne serait alors pas le fruit de son esprit. Ensuite, le fait qu'il puisse avoir utilisé une chronique comme source doit nous mettre en garde afin de ne pas chercher les sources de Jérôme seulement dans des histoires narratives. Certains savants sont partisans de la thèse arguant une forte dépendance de Jérôme à l'histoire entièrement perdue de Nicomaque Flavien (ca. 334-394) ou à la dite Kaisergeschichte ${ }^{54}$. Certes, ces idées ne sont pas invraisemblables, mais il faut toutefois se rendre compte qu'elle n'est pas

${ }^{52}$ R. W. Burgess, On the Date of the Kaisergeschichte. Classical Philology 90 (1995) 111-128, 126-127; ID., Jerome and the Kaisergeschichte. Historia 44 (1995) 349-369, 368. L'auteur situe la rédaction de la Kaisergeschichte en 357.

${ }^{53}$ Geppert, Quellen 69-75 a défendu l'idée que Socrate avait utilisé un texte de ce genre, sans pouvoir l'identifier à la Kaisergeschichte même.

${ }^{54}$ Flavien: S. RatTi, Jérôme et Nicomaque Flavien: sur les sources de la Chronique pour les années 357-364. Historia 46 (1997) 479-508; ID., Les sources de la chronique de 
la panacée qui résolvera le problème des sources de Jérôme - si ce problème peut trouver une solution satisfaisante.

(2) La chronique de 350 à 378 n'est pas la source de toutes les dates dans l'histoire de Socrate entre 350 et 378. Par exemple la dédicace de la S.Sophie (2.16.15-16), la mort d'Eudoxe (4.14.2) et celle d'Euzoios (4.35.4) ne peuvent pas y être attribuées. Comme pour le règne de Constantin, Socrate utilise de nombreuses sources qu'il intègre dans sa trame consulaire. Or, il se fait que ces sources supplémentaires ne sont plus identifiables ${ }^{55}$.

\subsubsection{Une chronique de 378 à 422 ou 439}

Une nouvelle césure se présente dans l'an $378^{56}$. Socrate situe la mort de Valens dans la quatrième année du 289ième olympiade et il lui accorde un règne de seize ans, qui serait selon lui de trois ans plus long que celui de son frère Valentinien (4.38.7). En assignant faussement un règne de treize ans à Valentinien, Socrate contredit ce qu'il déclarait quatre chapitres auparavant où Valentinien régnait correctement onze ans (4.31.6 ${ }^{57}$. Ces données contradictoires rendent évident le fait que l'historien se soit servi de deux sources distinctes pour ces deux passages. Les olympiades confirment cette conclusion. En effet, à partir de 378 les olympiades indiquées par Socrate présentent toutes un surplus d'un an par rapport à Eusèbe, et de deux ans par rapport à Jérôme, à l'exception de la dernière où le surplus monte à deux respectivement trois ans (tableau 2).

L'écart de deux ans par rapport à Jérôme s'explique par l'erreur initiale de cette chronique dans les années de règne: en assignant seize ans à Valens, l'éditeur a calculé deux ans de trop ${ }^{58}$. Ce surplus de deux ans dans

Jérôme pour les années 357-364: nouveaux éléments, in: L'historiographie de l'église des premiers siècles (Théologie historique 114). Paris 2001, 425-450; M. FEsTY, Le début et la fin des Annales de Nicomaque Flavien. Historia 46 (1997) 465-478. Kaisergeschichte: Burgess, Jerome.

55 Aussi pour ses autres livres, Socrate a sans doute utilisé des sources supplémentaires inconnues: voir Burgess, Studies 222-225 à propos de 1.39-40.

${ }_{56}^{56}$ Il ne nous est pas clair pourquoi L. JEEP, Quellenuntersuchungen zu den griechischen Kirchenhistorikern. Jahrbücher für classische Philologie 14 (1884) 53-178, 121, voit chez Socrate un changement de chronique en 395. L'idée de Burgess, Chronicle 197, que l'historien se sert d'une nouvelle chronique à partir de 383 , ne tient pas compte de toutes les indications disponibles.

${ }^{57} \mathrm{Il}$ semble que les copistes ont voulu corriger cette contradiction en corrigeant 11 en 13 (4.32.6), qui est la leçon des manuscrits grecs et de la traduction arménienne. Cassiodore et la traduction syriaque lisent 11, la leçon préférée par Hansen, Sokrates 267. 
les années de règne se traduit alors par un surplus similaire dans les olympiades (par rapport à Jérôme). Il y avait dès lors une chronique qui débutait en 378, qui acceptait le calcul hiéronymien pour les olympiades, et qui par une erreur initiale dans la longueur du règne de Valens, présentait un surplus de deux ans dans les olympiades par rapport à ce calcul hiéronymien.

La fin de cette chronique ne peut être déterminée de façon univoque. En faveur d'une fin en 422, on peut citer le fait que Socrate donne pour cette année-là l'olympiade (7.20.13) afin de marquer la fin de la guerre avec les Perses (421-422). C'est la seule fois qu'il propose l'olympiade pour dater un événement autre que la mort d'un empereur ou la fin d'un livre. Cette exception ne peut que difficilement être expliquée en termes d'importance de l'événement, car Socrate ne semble pas très hostile envers les Perses, qui n'ont qu'une place limitée dans son ouvrage ${ }^{59}$.

Un second élément soutient une fin en 422. Socrate semble avoir calculé lui-même la date en olympiades de 439 (7.48.8: Ol. 305.2). Or, cette dernière date présente un surplus d'un an par rapport à ce qui était usuel dans cette chronique (cf. tableau 2). Nous pouvons rendre compte de cette erreur à partir des autres données chronologiques que Socrate nous livre à la fin du septième livre. Selon lui, le dernier livre couvre trente-deux ans. C'est un an de trop, puisque Socrate a aussi inclus 408 alors que cette année était déjà comptée pour le règne d'Arcadius. Trente-deux ans après Ol. 279.2 (l'année de la mort d'Arcadius) est en effet Ol. 305.2 - un an de trop par le double calcul de $408^{60}$.

Si cette chronique se termine en 422 , Socrate a dû chercher ses informations historiques pour la période de 422 à 439 dans d'autres sources. Cette hypothèse n'est pas à exclure. Le récit de la victoire contre l'usurpateur Jean (7.22.20-23.10) est trop long pour provenir d'une chronique, tout comme la date de l'avènement de Valentinien III (7.25.23) ne présuppose pas nécessairement l'utilisation d'une chronique. Par la suite, Socrate ne donne plus des dates précises des événements de l'Empire; le mariage d'Eudoxie et de Valentinien III est même situé un an trop tôt, en 436 (7.44.1-3). En outre, Socrate reste bien informé sur les événements ecclésiastiques, qui, fort détaillés, ne proviennent sans doute pas d'une chronique.

58 Cette erreur restait jusqu'à présent énigmatique, cf. GEPPERT, Quellen 44-45; HANsEn, Sokrates li-lii; WaLlRafF, Kirchenhistoriker 157.

59 Cf. Socrate 3.19, 3.21, 7.19-22.

${ }^{60}$ Il est possible de résoudre d'autres erreurs de Socrate en acceptant qu'il compte quelque fois le début et la fin par deux fois, ef. Wallraff, Kirchenhistoriker 146 n. 42. On en trouve une illustration en 4.11.4, où Socrate compte 12 ans entre 358 et 368 . 
Il nous faut avouer que l'hypothèse selon laquelle Socrate aurait utilisé une chronique qui se termine en 422 nous semble défendable, mais qu'elle reste sans preuve solide. Si l'on n'accepte pas les arguments précités, tout porte à croire que la chronique a dû continuer jusqu'à la fin de l'ouvrage de Socrate, c'est-à-dire jusqu'en 439.

De nombreux parallèles avec Marcellinus Comes et avec la Chronique pascale, tantôt superficiels tantôt plus probants, nous permettent de mieux cerner cette source (tableau 7). Signalons plus particulièrement que la Chronique pascale partage avec Socrate les durées des règnes de Théodose I (seize ans) et d'Arcadius (quatorze ans) alors que la vaste majorité des sources leur accorde respectivement dix-sept ${ }^{61}$ et treize ans ${ }^{62}$. La spécificité de ce calcul devient claire si l'on se rend compte que le chiffre seize est obtenu en omettant la dernière année de Théodose (calcul eusébien), et celui de quatorze ans en comptant à partir 395 jusqu'en 408 - c'est-à-dire en comptant aussi la première année de Théodose II. Dans ce dernier cas, le chroniqueur n'avait pas respecté les règles habituelles et compté un an de trop pour Arcadius. Ces parallèles exceptionnels suggèrent leur dépendance à une source commune.

\begin{tabular}{|l|l|l|l|l|l|}
\hline Socrate & \multicolumn{1}{|c|}{ Marc. Com. } & Chron. Pasch. & \multicolumn{1}{|c|}{ Socrate } & \multicolumn{1}{|c|}{ Marc. Com. } & Chron. Pasch. \\
\hline $5.6 .2-6^{63}$ & ef. a. 380 & & $6.21 .1-2$ & a. 403 & \\
\hline $5.7 .1-11$ & ef. a. 380 & & $6.23 .7,7.1 .1$ & & p. 570.12 \\
\hline $5.10 .3-4$ & a. 381.2 & & $7.18 .24-25$ & & \\
\hline $5.14 .1-2$ & ef. a. 388 & & & & \\
\hline $5.14 .3-9$ & a. 389.1 & & $7.20 .1-13$ & ef. a. 422.4 & \\
\hline $5.16 .1-14$ & ef. $389.4^{64}$ & & 7.22 .20 & a. 423 & p. 580.4 \\
\hline
\end{tabular}

${ }^{61}$ Marcellinus Comes a. 395; Jean Malalas 13.37; Georgius Monachus, Chronique p. 561.20 (de Boor); Jacques d'Edesse p. 226 (CSCO 6); Chronicon 846 p. 159.1 (CSCO 5); Chronicon 724 p. 106.19 (CSCO 5); Chronique de Séert p. 268 (PO 5); Elias, Chronique p. 24 (CSCO 63).

${ }^{62}$ Marcellinus Comes a. 408; Jean Malalas 13.45; Georgius Monachus, Chronique. p. 592.2122 (de Boor); Jacques d'Edesse p. 228 (CSCO 6); Elias, Chronique p. 24 (CSCO 63). Une exception est Chronicon 846 p. 159.29 (CSCO 6), qui accorde 14 ans à Arcadius.

${ }^{63}$ Signalons une correction possible du texte de G. C. Hansen. À la page 277 1. 20 il écrit

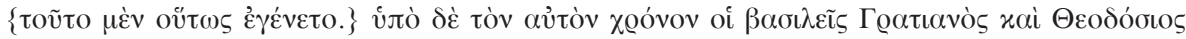

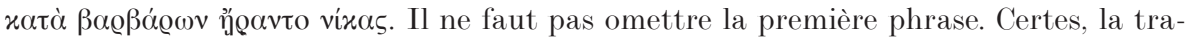
duction arménienne ne la donne pas, mais Socrate utilise des formules identiques en

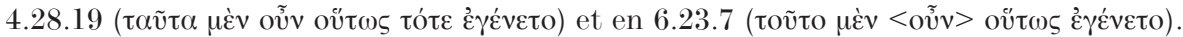

${ }^{64}$ La datation de l'événement, la destruction du Sérapéum, varie: Socrate le situe en 388 , Marcellinus en 389, alors qu'en vérité il avait lieu en 391. Socrate peut aussi dépendre de témoins oculaires dans ce passage. 


\begin{tabular}{|l|l|l|l|l|l|}
\hline \multicolumn{1}{|c|}{ Socrate } & \multicolumn{1}{|c|}{ Marc. Com. } & Chron. Pasch. & \multicolumn{1}{|c|}{ Socrate } & \multicolumn{1}{|c|}{ Marc. Com. } & Chron. Pasch. \\
\hline $5.18 .12-14$ & cf. a. 391 & & $7.23 .1-10$ & a. $424-425.1$ & $\begin{array}{l}\text { cf. p. } 580 . \\
13-15\end{array}$ \\
\hline $5.25 .1-16$ & ef. a. $393-394$ & & 7.25 .23 & a. 425 & \\
\hline 5.26 .6 & & $\begin{array}{l}\text { cf. p. } 565 . \\
11-13\end{array}$ & $7.31 .1-6$ & a. 429 & \\
\hline $6.1 .3-7^{65}$ & a. 395.2 & p. $566.1-3$ & $7.33 .1-5$ & a. 431.2 & \\
\hline $6.6 .38-40$ & a. 401.3 & p. $567.12-20$ & $7.39 .1-10^{66}$ & a. 433 & p. 582.4-6 \\
\hline 6.18 .1 & a. 403.2 & af. p. $568.14-$ & $7.44 .1-3^{67}$ & a. 437 & p. $582.13-17$ \\
\hline $6.18 .18^{68}$ & a. 404 & 17 & a. 438 & \\
\hline
\end{tabular}

tableau 7

Il est difficile de tirer des conclusions infaillibles de ce tableau. Il est connu qu'à partir des années 384 jusqu'en 469 Marcellinus Comes et la Chronique pascale puisent d'une même source ${ }^{69}$. Du tableau on peut conclure qu'il y a des parallèles entre cette chronique commune et Socrate (par exemple Socr. 6.1.3-7, 6.6.38-40, 7.22.20). Cela suggère que cette chronique-là ait utilisé soit Socrate soit la source de Socrate. La seconde option, une chronique copiant une autre, nous semble plus probable, sans que nous puissions la démontrer.

Un usage immédiat de Socrate par la Chronique pascale est exclu comme nous l'avons déjà dit. Par contre, Marcellinus Comes peut s'être servi de Socrate, à côté de la source commune qu'il partage avec la Chronique pascale. Par exemple l'histoire de l'évêque Antoninus qui s'en prit aux macédoniens (Marcellinus Comes a. 429, Socr. 7.31.1-6) ne trouve aucun parallèle dans la Chronique pascale et peut provenir immédiatement de Socrate ${ }^{70}$.

La source de Socrate peut donc avoir été une source de la chronique commune qu'utilisent Marcellinus et la Chronique pascale pour les années 384-469. Marcellinus Comes peut, en plus, s'être servi indépendemment de l'Histoire ecclésiastique de Socrate.

65 Cf. B. Croke, Count Marcellinus and his Chronicle. Oxford 2001, 207.

66 Socrate et la Chronique pascale divergent sur la date du miracle dans une église novatienne de Constantinople: 17 respectivement 21 août. Socrate dépend peut-être de témoins oculaires dans ce passage.

${ }^{67}$ La Chronique pascale donne une date précise, alors que Socrate ne donne que l'année.

68 L'information de la Chronique pascale est un peu plus ample par rapport à Socrate.

69 L'édition de Mommsen, Chronica minora: Vol. 2 64-90 laisse les parallèles commencer en 395, mais voir Croke, Count Marcellinus 179-184.

${ }^{70}$ Cet exemple montre que, à notre avis, B. Croke a trop rapidement écarté la possibilité que Marcellinus ait utilisé immédiatement Socrate (Count Marcellinus 206). 


\section{Conclusion}

Nous nous sommes beaucoup éloignés de l'unique chronique dans laquelle Socrate aurait puisé la majorité de ses données. Quatre chroniques et deux consularia ont été identifiés comme sources de l'historien. Ce constat a des conséquences pour la méthode de Socrate, mais aussi pour notre perception de la littérature chronographique de l'époque, tout comme pour les chroniques de Jérôme et de Marcellinus Comes et la Chronique pascale.

(1) Sur le plan méthodologique, nous pouvons avancer que Socrate est un fainéant quand il se trouve aux prises avec des chroniques ${ }^{71}$. Bien qu'il se trouve face à une lacune de deux ans entre les deux consularia dont il dispose, il n'a pas cherché une meilleure liste. Lorsqu'une chronique contredit l'autre, il n'intervient pas. Il s'arrête à copier simplement les indications chronologiques de ses sources sans les unifier dans un système propre. Il ne nous faut pas dresser à nouveau la liste des erreurs et des contradictions; celles-ci nous ont servi afin de reconstruire les sources de Socrate ${ }^{72}$.

Or, sa méthode semble claire: les consularia forment le squelette chronologique de son ouvrage, dans lequel il essaie d'intégrer les données chronographiques des autres sources. Par ailleurs, la plupart des informations supplémentaires proviennent de chroniques. À côté de celles-ci, des ouvrages comme celui d'Eutrope ou la Vie de Constantin d'Eusèbe ont fourni d'autres données et des dates.

(2) Il nous faut accepter l'existence d'une multitude de chroniques et de consularia à Constantinople au quatrième et cinquième siècle. Pour défendre l'idée d'une seule chronique officielle dans chaque ville importante, résumée et publiée de temps en temps, Socrate n'offre aucune preuve. Au contraire, son usage de plusieurs chroniques et consularia falsifie cette hypothèse, pour laquelle il nous manque d'ailleurs tout indice convaincant ${ }^{73}$. Les ouvrages qui nous sont parvenus ne sont qu'une mince partie de ce foisonnement des chroniques. Les ombres de deux de ces chroniques perdues se retrouvent dans l'ouvrage de Socrate: une chronique de 350 à 378

71 Le sévère jugement d'E. Schwartz, comme quoi Socrate n'aurait fait qu'amasser ses sources (Zur Geschichte des Athanasius, in: Gesammelte Schriften 3. Berlin 1959, 117 n. 1) se révèle vrai, au moins en ce qui concerne l'usage des chroniques. Theresa Urbainczyk, Socrates of Constantinople. Historian of Church and State. Michigan 1997, 170, est trop optimiste sur la méthode de Socrate.

72 Signalons que nous ne savons pas comment expliquer l'erreur finale de Socrate, qui prétend que son histoire couvre 140 ans, alors qu' en vérité il ne s'agit que de 133 ans.

73 Cf. Burgess, Chronicle 178-186. 
que Socrate semble avoir partagée avec Jérôme et avec la Chronique pascale, et une chronique constantinopolitaine de 378 à 422 ou 439, qui était incorporée dans la source commune de Marcellinus Comes et de la Chronique pascale.

(3) La reconstruction hypothétique de ces deux dernières chroniques, si on l'accepte, comporte les conséquences suivantes pour Jérôme, Marcellinus Comes et la Chronique pascale. Elles doivent évidemment rester en large mesure hypothétiques.

(3.1) À part des sources narratives, la chrônique de Jérôme dépend aussi d'autres chroniques, dont il emprunte plusieurs éléments. Nous avons proposé l'existence d'une chronique allant de 350 à 378, que Jérôme partage avec Socrate. De cet ouvrage, il a peut-être pris le calcul des années de règne, les olympiades, la fin en 378, et évidemment quelques notices.

(3.2) Il est, ensuite, probable que Marcellinus Comes a consulté Socrate indépendamment pour quelques notices. En même temps, il se servait d'une version ultérieure d'une chronique que Socrate utilisait pour les années de 378 à 422 ou 439 .

(3.3) La Chronique pascale semble avoir dû intégrer deux chroniques indépendantes, l'une terminant et l'autre débutant en 378. Ceci s'avère une cause possible de la confusion présente dans la chronique à cet endroit. En effet, Valentinien se voit accordé quatorze ans de règne et sa mort est située en 378 au lieu de 375 (p. 555.20). Par ailleurs, la chronique invente un corègne de Valens, Gratien et Théodose I. Cette confusion peut être due à un essai maladroit d'unification de deux chroniques différentes, qui comme nous l'avons $\mathrm{vu}$, donnaient des indications contradictoires. 
